\title{
Permissive fluid volume in adult patients undergoing extracorporeal membrane oxygenation treatment
}

Hyoungnae Kim,2, Jin Hyuk Paek ${ }^{3}$, Joo Han Song ${ }^{4}$, Hajeong Lee ${ }^{5}$, Jong Hyun Jhee ${ }^{6}$, Seohyun Park ${ }^{2}$, Hae-Ryong Yun², Youn Kyung Kee', Seung Hyeok Han², Tae-Hyun Yoo ${ }^{2}$, Shin-Wook Kang ${ }^{2}$, Sejoong Kim ${ }^{3 *}$ and Jung Tak Park ${ }^{2 *}$

\begin{abstract}
Background: Extracorporeal membrane oxygenation (ECMO) is a cardiorespiratory support technique for patients with circulatory or pulmonary failure. Frequently, large-volume fluid resuscitation is needed to ensure sufficient extracorporeal blood flow in patients initiating ECMO. However, excessive overhydration is known to increase mortality in critically ill patients. Therefore, in order to define a tolerant volume range in patients undergoing ECMO treatment, the association between cumulative fluid balance (CFB) and outcome was evaluated in patients undergoing ECMO.
\end{abstract}

Methods: This retrospective multicenter cohort study was conducted with 723 patients who underwent ECMO in three tertiary care hospitals between 2005 and 2016. CFB was calculated as total fluid input minus total fluid output during the first 3 days from ECMO initiation. The patients were divided into groups that initiated ECMO owing to cardiovascular disease (CVD)-related or non-cardiovascular disease (non-CVD)-related causes. The primary endpoint was mortality within 90 days after ECMO commencement.

Results: Totals of 406 and 317 patients were included in the CVD and non-CVD groups, respectively. In the CVD group, the mean age was $58.4 \pm 17.7$ years, and $68.2 \%$ were male. The mean age was $55.7 \pm 15.7$ years, and $65.3 \%$ were male in the non-CVD group. The median CFB values were 64.7 and $53.5 \mathrm{ml} / \mathrm{kg}$ in the CVD and non-CVD groups, respectively. Multivariable analysis using Cox proportional hazards models revealed a significantly increased risk of 90-day mortality in patients with higher CFB values in both the CVD and non-CVD groups. However, the risks were elevated only in the two CFB quartile groups with the largest CFB amounts. Cubic spline models showed that mortality risk began to increase significantly when CFB was $82.3 \mathrm{ml} / \mathrm{kg}$ in the CVD group. In patients with respiratory diseases, the mortality risk increase was significant for those with CFB levels above $189.6 \mathrm{ml} / \mathrm{kg}$.

Conclusions: Mortality risk did not increase until a certain level of fluid overload was reached in patients undergoing ECMO. Adequate fluid resuscitation is critical to improving outcomes in these patients.

Keywords: Acute respiratory distress syndrome, Cardiogenic shock, Extracorporeal membrane oxygenation, Fluid balance, Mortality

\footnotetext{
*Correspondence: sejoong2@snu.ac.kr; jtpark@yuhs.ac

${ }^{3}$ Department of Internal Medicine, Seoul National University Bundang Hospital, 82, Gumi-ro 173 Beon-gil, Bundang-gu, Seongnam, Gyeonggi-do 13620, Republic of Korea

${ }^{2}$ Department of Internal Medicine, Institute of Kidney Disease Research,

Yonsei University College of Medicine, 50-1 Yonsei-ro, Seodaemun-Gu, Seoul

03722, Republic of Korea

Full list of author information is available at the end of the article
}

(c) The Author(s). 2018 Open Access This article is distributed under the terms of the Creative Commons Attribution 4.0 International License (http://creativecommons.org/licenses/by/4.0/), which permits unrestricted use, distribution, and reproduction in any medium, provided you give appropriate credit to the original author(s) and the source, provide a link to the Creative Commons license, and indicate if changes were made. The Creative Commons Public Domain Dedication waiver (http://creativecommons.org/publicdomain/zero/1.0/) applies to the data made available in this article, unless otherwise stated. 


\section{Background}

Extracorporeal membrane oxygenation (ECMO) is a salvage therapy for patients with severe respiratory and heart failure [1-4]. Patients frequently require large-volume fluid resuscitation during the initial phases of ECMO treatment in order to maintain a sufficient amount of vascular blood drainage for extracorporeal blood flow [5-7]. This need for liberal fluid infusion during ECMO treatment is further exacerbated by the fact that most patients undergoing ECMO treatment are in an intravascular hypovolemic state aggravated by systemic capillary leakage $[8,9]$. In addition to the inevitable large-volume fluid resuscitation, administration of blood products for bleeding events accompanying ECMO implantation and reduced urine volume caused by concomitant acute kidney injury (AKI) also play a part in the aggravation of fluid overload in patients undergoing ECMO [10-14]. Furthermore, excessive positive fluid balance during intensive care unit (ICU) stay is reported to affect outcome, and a positive fluid balance was found to increase the risk of mortality in patients with septic shock [15-17]. In addition, large-volume intravenous fluid therapy was associated with more cardiac arrest events and increased pulmonary edema in patients resuscitated from cardiac arrest events [18], and excessive volume overload was found to be linked to poor survival, even in patients undergoing ECMO therapy $[19,20]$. Therefore, although large-volume resuscitation is necessary in most patients initiating ECMO, excessive volume overload may lead to poor outcomes. The clinically significant volume overload threshold has not yet been established.

Cumulative fluid balance (CFB) has been widely used as a surrogate marker of fluid therapy during ICU treatment. Higher CFB values after ICU admission were found to increase the risk of AKI development [21]. In addition, CFB has been reported to be associated with worse clinical outcomes in patients with sepsis [15, 22], acute lung injury [23, 24], and cancer [25]. Therefore, the association between CFB during the initial phase of ECMO treatment and outcome was evaluated, and a clinically significant threshold level of CFB that affects outcome was investigated.

\section{Methods}

\section{Patient selection}

Patients who received ECMO at Yonsei University Health System, Seoul National University Hospital, and Seoul National University Bundang Hospital from January 2005 to May 2016 were initially screened $(n=1499)$. Patients who met the following criteria were excluded ( $n=776)$ : (1) age under 18 years, (2) ECMO support for less than $24 \mathrm{~h}$, (3) death within 3 days from ECMO initiation, (4) switching of ECMO modality, (5) end-stage renal disease with dialysis at admission, (6) transfer after initiating ECMO in other hospitals, and (7) missing data. As a result, 406 patients who underwent ECMO for cardiovascular causes (cardiovascular disease [CVD] group) and 317 patients who underwent ECMO for non-cardiovascular causes (non-CVD group) were included in the final analysis (Fig. 1). Although myocarditis is caused by infection, patients with myocarditis were included in the CVD group on the basis of pathophysiologic considerations. The study protocol was approved by the institutional review boards of Yonsei University Health System and Seoul National University Bundang Hospital. Informed consent was waived by the institutional review boards owing to the retrospective study design.

\section{Data collection}

The patients' demographic and laboratory data at the time of ECMO initiation were retrieved from electronic medical records. The data included age, sex, height, weight, underlying medical conditions, cause of ECMO, mode of ECMO (venoarterial [VA]-ECMO or venovenous [VV]-ECMO), ECMO settings, and total fluid input and output on each day of ECMO therapy. Laboratory data, including white blood cell count, hemoglobin, sodium, potassium, bicarbonate, albumin, bilirubin, C-reactive protein, blood urea nitrogen, creatinine, and

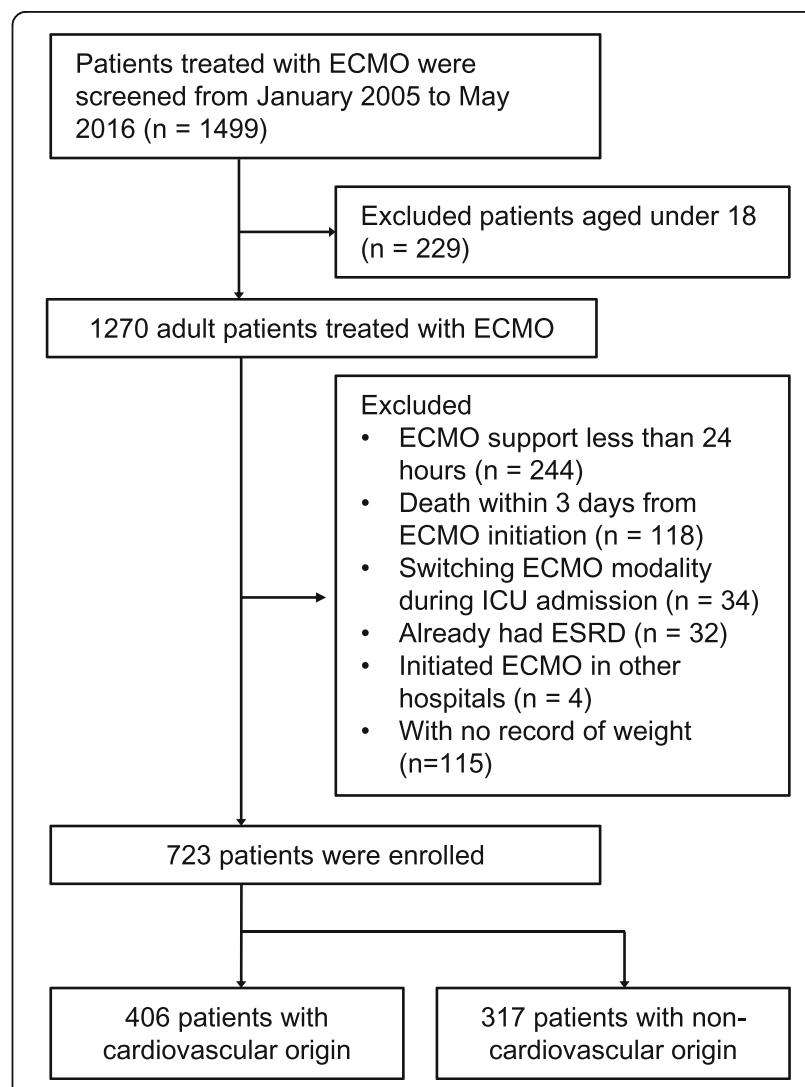

Fig. 1 Flowchart of participant enrollment. ECMO Extracorporeal membrane oxygenation, ESRD End-stage renal disease 
arterial blood gas analysis on the day of ECMO implantation, were also collected.

Acute Physiology and Chronic Health Evaluation (APACHE) II scores were calculated on the basis of medical records. The ENCOURAGE (Prediction of cardiogenic shock outcome for AMI patients salvaged by VA-ECMO) and PRESERVE (Predicting death for severe ARDS on VV-ECMO) scores were also calculated for patients in the CVD and non-CVD groups, respectively $[26,27]$. The Modification of Diet in Renal Disease equation was used to determine the estimated glomerular filtration rate (eGFR) [28]. Baseline serum creatinine was defined as the average serum creatinine within 3 months prior to ICU admission. For those whose baseline creatinine levels were not obtainable, urine output was used for AKI diagnosis. Development of AKI within $72 \mathrm{~h}$ of ECMO implantation was evaluated using the Kidney Disease: Improving Global Outcomes (KDIGO) AKI criteria [29]. The amount of fluid administered was decided by the intensive care specialists. Crystalloid fluids were used to maintain fluid therapy for all patients. The low-chloride fluid resuscitation strategy had not been widely practiced in the institutions during the study period. Cumulative input was defined as total administration of fluid within 3 days of ECMO initiation, which was divided by patients' body weight at the time of ECMO initiation. In addition, cumulative total output was defined as the sum of all fluid drainage from patients, and cumulative urine output as the sum of the total amount of urine during 3 days from ECMO initiation divided by body weight. CFB was defined as cumulative input minus cumulative total output. Daily fluid balance before ECMO was defined as total fluid input minus total output from ICU admission to ECMO initiation, which was divided by ICU length of stay (LOS) and patients' body weight. If patients were admitted to the ICU after surgery, fluid balance during surgery was also included in daily fluid balance before ECMO.

\section{ECMO protocol}

The decision to initiate ECMO therapy was made by the treating intensive care specialist or the attending cardiothoracic surgeons. Indication for ECMO was classified into two categories. Indications for ECMO in the CVD group were as follows:

1. Postcardiotomy status

2. Acute cardiogenic shock for coronary arterial disease

3. Acute myocarditis

4. Bridge to cardiac transplant for decompensated heart failure

5. Post-cardiac arrest status
Indications for $\mathrm{ECMO}$ in the non-CVD group were as follows:

1. Acute respiratory distress syndrome (ARDS) of any cause

2. Bridge to lung transplant

3. Miscellaneous or unknown origin

Cannulations for all ECMO supports were performed by cardiovascular surgeons with limited cut-down using the Seldinger technique [30]. Either the CAPIOX EBS (Terumo Co., Ltd., Tokyo, Japan) or the QUADROX PLS (Maquet GmbH, Rastatt, Germany) system was used in all patients. The sweep gas flow was set to maintain a partial pressure of carbon dioxide target of 35-45 $\mathrm{mmHg}$ under lung rest ventilation strategy. The target ECMO blood pump speed was 3.0-4.5 L/min. Systemic anticoagulation with unfractionated heparin was titrated to maintain activated clotting times (ACT) between 180 and $220 \mathrm{~s}$ and between 160 and $180 \mathrm{~s}$ for VA-ECMO and VV-ECMO, respectively. ACT was measured using STA R MAX $^{\circ}$ hemostasis analyzer (Diagnostica Stago, Inc., Parsippany, NJ, USA). Hemolysis markers, such as free plasma hemoglobin and haptoglobin, were monitored every day. Careful inspections were made by the intensive care specialist to determine the cause of hemolysis when plasma hemoglobin levels were greater than $50 \mathrm{mg} / \mathrm{dl}$. ECMO circuit change was performed when ECMO-related technical problems were suspected as the cause of hemolysis. Red blood cell (RBC) transfusion was performed to maintain hemoglobin target between 10 and $11 \mathrm{~g} / \mathrm{dl}$. Antithrombin (AT) replacement was performed when AT activities were less than $70 \%$. Other specific ECMO run protocols are delineated in Additional file 1: Table S1.

\section{Continuous renal replacement therapy protocol}

In the event of the development of AKI, the decision to start continuous renal replacement therapy (CRRT) was made by the attending nephrologist. Generally, CRRT was applied to patients with AKI who had uncontrolled fluid overload, hyperkalemia, or metabolic acidosis. Continuous venovenous hemodiafiltration was applied using Prisma (Gambro Co., Ltd., Hechingen, Germany), Prismaflex (Gambro Co., Ltd.), or multiFiltrate (Fresenius Medical Care GmbH, Bad Homburg, Germany) machines. Biocompatible AN69 ST membranes were used for Prisma and Prismaflex, and polysulfone membranes were used for multiFiltrate. Bicarbonate-containing replacement fluid was administered by the predilution method. CRRT machines were routinely applied via separate lines from the ECMO circuit. However, when additional venous access was not obtainable, CRRT was performed over the same circuit with ECMO. In these 
cases, CRRT was applied between ECMO pump and oxygenator to avoid air entrainment.

\section{Clinical outcome}

The patients were followed until their last visit at our centers or death. The primary endpoint was death from any cause within 90 days after ECMO initiation. Survival data were collected through electronic medical records of the hospital and outpatient clinics.

\section{Statistical analysis}

Patients in each group were categorized into quartiles according to the CFB of each group. Continuous variables were expressed as mean $\pm \mathrm{SD}$. Comparisons of continuous variables were conducted with one-way analysis of variance, and linear trend was analyzed among groups. The normality of the distribution was analyzed using the Kolmogorov-Smirnov test. Variables not normally distributed were expressed as median and IQR and compared with the Jonckheere-Terpstra test. Categorical variables were expressed as percentages and compared with the chi-square test. Cumulative survival curves were derived using the Kaplan-Meier method, and statistical differences were analyzed using the log-rank test. To evaluate the association between outcomes and clinical parameters, a Cox proportional hazards model was used, and data were expressed as HR with 95\% CI. This model included propensity scores. The propensity score was obtained by using a multivariate logistic regression analysis to determine whether the CFB of each patient was higher than the median amount. The covariates for adjustment were selected using a stepwise procedure when the $P$ value for each variable was less than 0.2 . All variables, except those that were already included in the APACHE II score, were included for propensity score calculation. Those who were lost to follow-up were treated as censored in the survival analysis. Cubic spline curve analysis was conducted as previously described by Canaud et al. [31]. The Cox model for 90-day mortality was used, and cubic spline curves had four equally distributed knots. The threshold of CFB was defined as the point where the lower limit of the 95\% CI was higher than 1.0. Analyses were conducted for the CVD and non-CVD groups. Additional evaluations were also conducted after dividing the non-CVD group into a respiratory group and an others group. The respiratory group was defined as patients who underwent ECMO for lung transplant, ARDS, or other pulmonary diseases. All other patients in the non-CVD group were defined as the others group. Statistical significance was defined as $P<0.05$. All analyses were conducted using SPSS version 23.0 software (IBM, Armonk, NY, USA) and R language (version 3.3.1; R Foundation for Statistical Computing, Vienna, Austria).

\section{Results}

Baseline characteristics of patients

The baseline characteristics of the patients in the CVD group are presented in Table 1 . The mean age was 58.4 \pm 17.7 years, and $68.2 \%$ were male. The most common cause of ECMO implantation was nonoperative CVD, and $98 \%$ of patients underwent VA-ECMO. The median CFB was $64.7 \mathrm{ml} / \mathrm{kg}$, whereas the median daily fluid balance throughout the entire period of ECMO was $26.2 \mathrm{ml} / \mathrm{kg} / \mathrm{d}$. The median duration of ECMO treatment was 3 days. The age of the patients tended to increase in patients with higher CFB quartiles. Body weight tended to decrease in higher CFB quartiles, but body mass index (BMI) was comparable among the quartiles. In the non-CVD group, the mean age was $55.7 \pm 15.7$ years, and $65.3 \%$ of patients were male. Among these patients, $201(63.8 \%)$ patients received ECMO treatment for respiratory disease, and VA-ECMO was applied for $23 \%$ of the patients. The median CFB was $53.5 \mathrm{ml} / \mathrm{kg}$, and median daily fluid balance during the entire ECMO period was $15.9 \mathrm{ml} / \mathrm{kg}$. Patients' weight and BMI were comparable among the CFB quartiles. However, APACHE II scores tended to increase in patients with higher CFB quartiles (Table 2). In the non-CVD group, there were no significant differences in Charlson comorbidity index $(P=0.616)$, APACHE II score $(P=0.302)$, and CFB $(P=0.206)$ between VA-ECMO- and VV-ECMO-treated patients. Regarding the resuscitation volume differences during the study duration, further analyses were made by categorizing the patients into three groups according vintage: 2005-2008, 2009-2012, and 2013-2016. When the CFB quartiles were compared among the vintage groups, no statistical differences were found (Additional file 2: Figure S1).

\section{Outcome of patients}

In the CVD group, AKI occurred in 306 (75.4\%) patients and CRRT was commenced in 127 (31.3\%) patients during the initial 3 days of ECMO initiation. The occurrence rate of AKI tended to increase in quartiles with higher CFB values. In addition, in the CVD group, median ICU LOS was 8 days. A total of 207 deaths (51.0\%) occurred within 90 days of ECMO initiation, and the mortality rate tended to increase in higher CFB quartiles $(P<0.001)$. In the non-CVD group, 184 $(70.0 \%)$ patients underwent AKI, and CRRT was applied in $92(29.0 \%)$ patients during first 3 days of ECMO commencement. Both AKI occurrence $(P=0.011)$ and CRRT application rates $(P=0.011)$ tended to increase in patients with higher CFB. Patients treated with VA-ECMO had a significantly higher incidence of AKI than those treated with VV-ECMO $(P=0.014)$. The median ICU LOS was 16 days. During the 90 days of ECMO initiation, a total of $209(65.9 \%)$ deaths occurred (Table 3). 


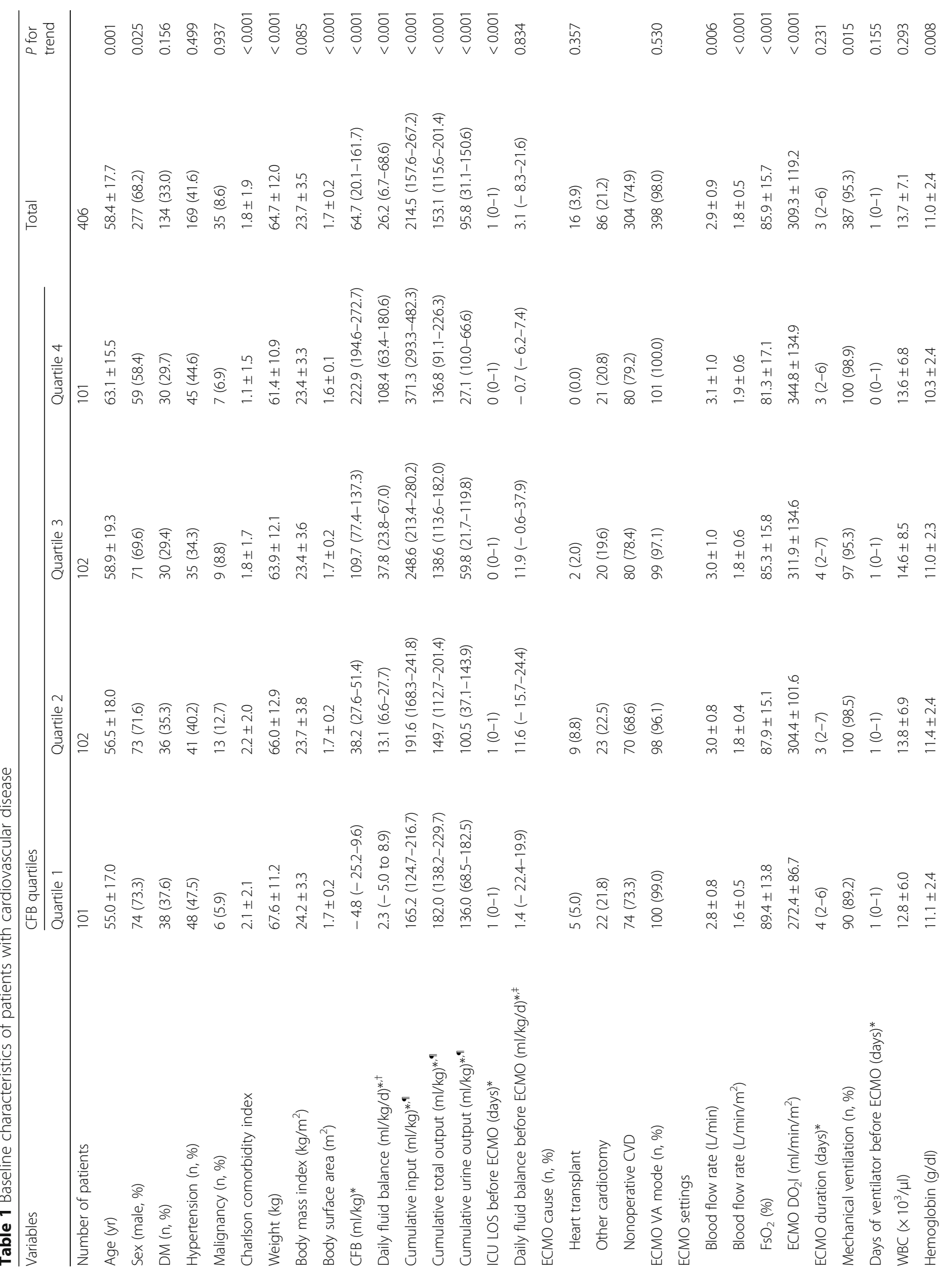




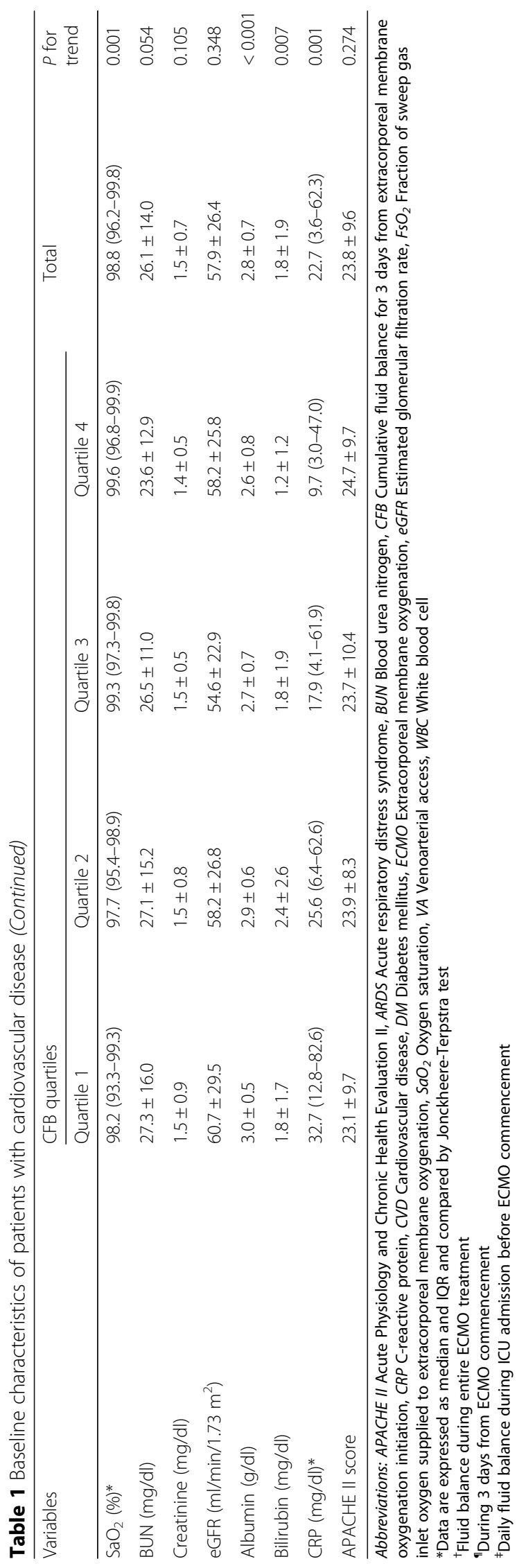




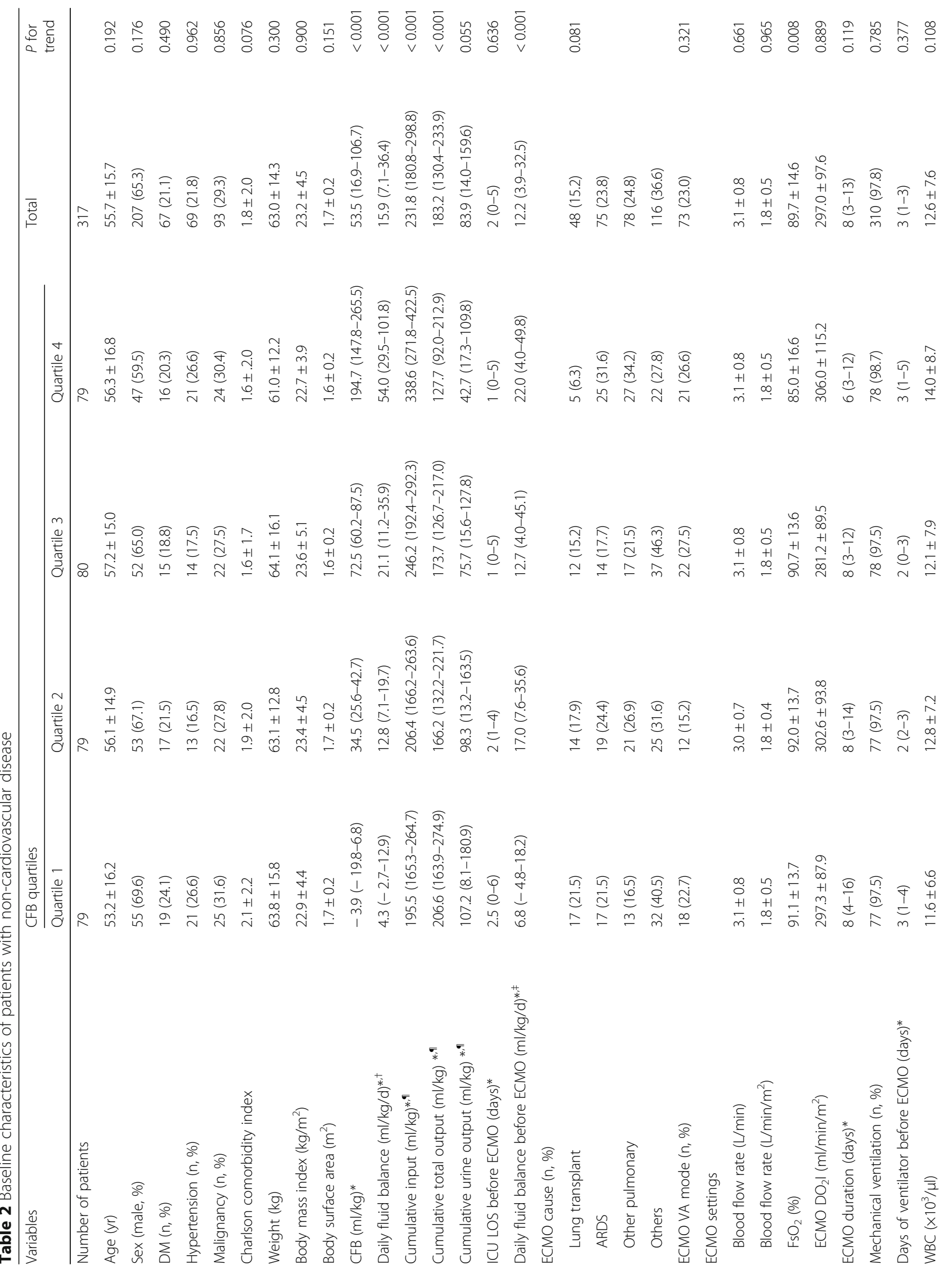




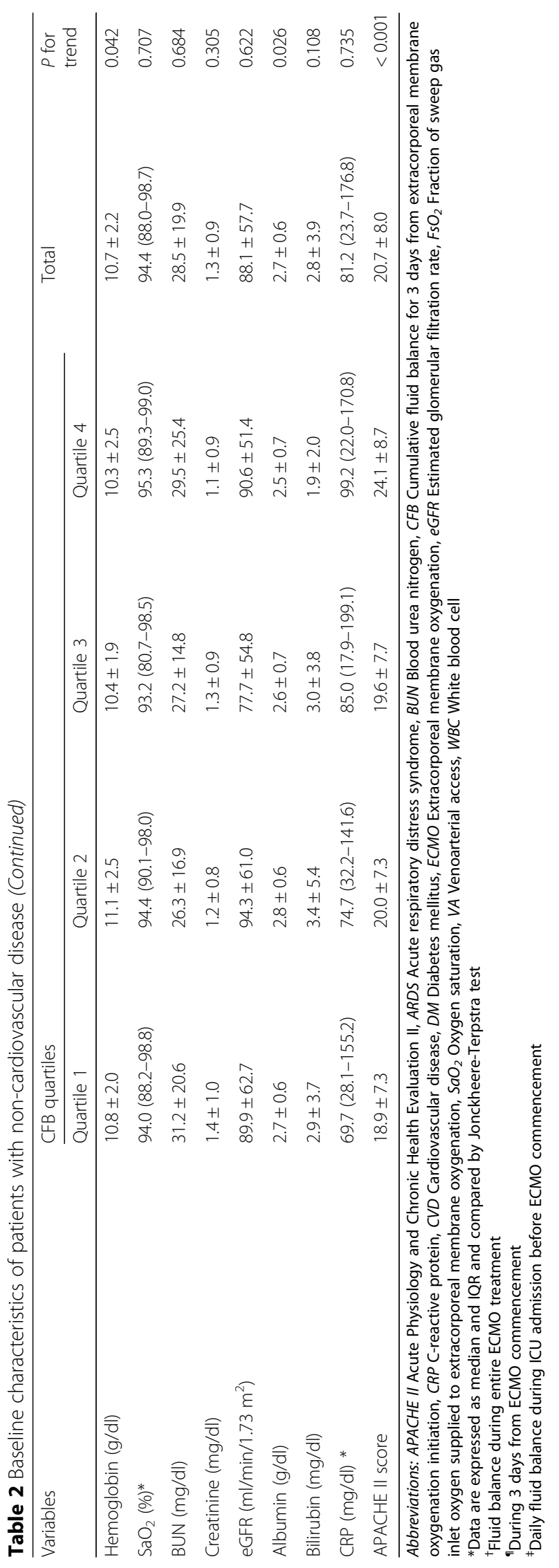


Table 3 Outcome of patients according to cumulative fluid balance quartiles

\begin{tabular}{|c|c|c|c|c|c|c|}
\hline \multirow[t]{2}{*}{ Variables } & \multicolumn{4}{|c|}{ CFB quartiles } & \multirow[t]{2}{*}{ Total } & \multirow{2}{*}{$\begin{array}{l}P \text { for } \\
\text { trend }\end{array}$} \\
\hline & Quartile 1 & Quartile 2 & Quartile 3 & $\overline{\text { Quartile } 4}$ & & \\
\hline \multicolumn{7}{|l|}{ Cardiovascular disease } \\
\hline Incident AKI (n, \%) & $60(59.3)$ & $76(74.4)$ & $86(84.3)$ & $84(83.1)$ & $306(75.4)$ & $<0.001$ \\
\hline CRRT treatment $(n, \%)^{*}$ & $26(25.7)$ & $24(23.5)$ & $48(47.1)$ & $29(28.7)$ & $127(31.3)$ & 0.114 \\
\hline Days of CRRT before ECMO (days) ${ }^{\dagger}$ & $0(0-1)$ & $0(0-1)$ & $1(0-1)$ & $0(0-2)$ & $1(0-1)$ & 0.082 \\
\hline ICU LOS (days) $)^{\dagger, \ddagger}$ & $10(5-16)$ & $10(6-18)$ & $7(3-14)$ & $7(4-14)$ & $8(4-15)$ & 0.014 \\
\hline 90-day mortality $(n, \%)$ & $35(34.7)$ & $43(42.2)$ & $71(69.6)$ & $58(57.4)$ & $207(51.0)$ & $<0.001$ \\
\hline \multicolumn{7}{|l|}{ Non-cardiovascular disease } \\
\hline Incident AKI (n, \%) & $47(68.1)$ & $34(53.1)$ & $54(76.1)$ & $49(83.1)$ & $184(70.0)$ & 0.011 \\
\hline CRRT treatment $(n, \%)^{*}$ & $17(21.5)$ & $16(20.3)$ & $32(40.0)$ & $27(34.2)$ & $92(29.0)$ & 0.011 \\
\hline Days of CRRT before ECMO (days) ${ }^{\dagger}$ & $1(0-2)$ & $1(1-2)$ & $2(1-2)$ & $1(1-2)$ & $3(1-3)$ & 0.483 \\
\hline ICU LOS (days) & $18(10-33)$ & $17(11-28)$ & $13(8-31)$ & $17(6-40)$ & $16(9-32)$ & 0.239 \\
\hline 90-day mortality (n, \%) & $49(62.0)$ & $43(54.4)$ & $63(78.8)$ & $54(68.4)$ & 209 (65.9) & 0.069 \\
\hline
\end{tabular}

Abbreviations: AKI Acute kidney injury, CFB Cumulative fluid balance, CRRT Continuous renal replacement therapy, ECMO Extracorporeal membrane oxygenation, ICU Intensive care unit, LOS Length of stay

*Continuous renal replacement therapy imitation during the first 3 days of ECMO commencement

${ }^{\dagger}$ Data are expressed as median and IQR and compared by Jonckheere-Terpstra test

${ }^{\ddagger} \mathrm{LOS}$ was presented in patients who survived at 30th day from ECMO initiation

\section{Relationship between CFB quartile groups and mortality}

The Kaplan-Meier curves revealed that the cumulative survival rates of CFB quartiles $3(P<0.001)$ and $4(P<$ $0.001)$ were significantly lower than those of CFB quartiles 1 and 2 in the CVD group (Fig. 2a). The non-CVD group was further divided into those initiating ECMO for respiratory failure (respiratory group) and other causes (others group). The characteristics of the respiratory group are presented in Additional file 1: Table S2. In patients in the respiratory group, the cumulative survival rate of CFB quartile 4 was significantly lower than quartile $1(P=0.047)$ (Fig. 2b). In addition, in the others group, CFB quartile 4 showed a significantly lower cumulative survival rate than quartile $1(P=0.004)$ and quartile $2(P=0.001)$ (Fig. 2c). When patients in the non-CVD group were divided into those treated with VA-ECMO or VV-ECMO, those treated with VA-ECMO had significantly lower cumulative survival rates than those treated with VV-ECMO $(P=0.002)$.

In addition, when the association between CFB and mortality was further evaluated using multivariate Cox proportional hazards regression analyses, in the CVD group, the risk of mortality significantly increased in patients in CFB quartiles 3 (HR, 2.58; 95\% CI, 1.62-4.11; $P$ $<0.001)$ and $4(\mathrm{HR}, 2.11 ; 95 \% \mathrm{CI}, 1.26-3.54 ; \mathrm{P}=0.004)$ compared with that of patients in CFB quartile 1. However, the mortality risk did not show a significant increase among patients in CFB quartile 2. Similarly, in
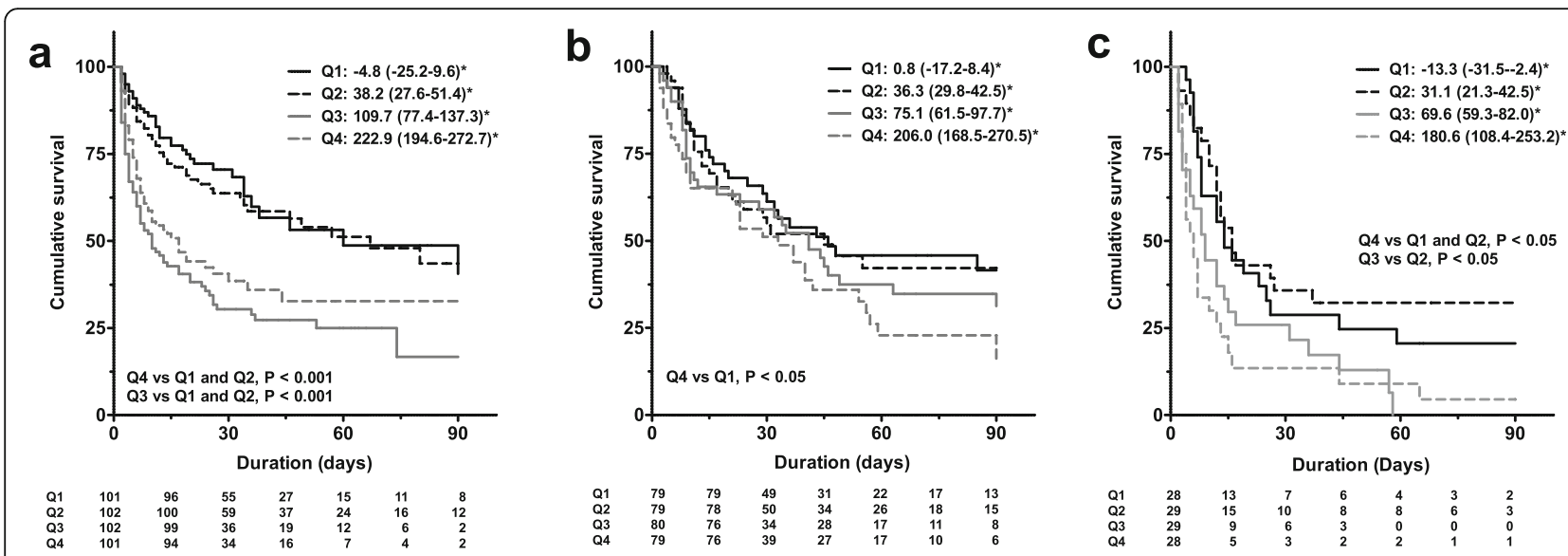

Fig. 2 Kaplan-Meier (KM) plots for 90-day mortality in each cumulative fluid balance quartile. a KM plots representing patients with cardiovascular disease. $\mathbf{b}$ KM plots representing patients with respiratory disease. c KM plots representing patients with other disease. *Median and IQR of cumulative fluid balance $(\mathrm{ml} / \mathrm{kg})$ for each quartile 
the non-CVD group, the mortality risk of CFB quartiles 3 (HR, 1.66; 95\% CI, 1.06-2.59; $P=0.026)$ and 4 (HR, $1.69,95 \% \mathrm{CI}, 1.05-2.72 ; \quad P=0.03)$ was significantly higher than quartile 1 , whereas mortality risk did not significantly differ between CFB quartiles 1 and 2 (Table 4). These findings imply that a CFB below the level of CFB quartile 3 in both the CVD and non-CVD groups may not increase the risk of mortality. When CFB was considered as a continuous variable, log-transformed CFB was independently associated with 90-day mortality in both the CVD group (HR, 1.76; 95\% CI, 1.37-2.27; $P<0.001)$ and the non-CVD group (HR, 1.46; 95\% CI, 1.17-1.83; $P<0.001$ ) (Table 5).

No significant interactions between APACHE II scores and CFBs were found in both the CVD and non-CVD groups $(P=0.521$ for CVD group, $P=0.166$ for non-CVD group). When further adjustments were made for daily fluid balance before ECMO commencement, the relationship between CFB and risk of mortality was preserved in both groups. In addition, cumulative input was independently associated with 90-day mortality even after adjustments were made for cumulative total output and urine output (Table 5).

When multivariate Cox models were analyzed with adjustment for ENCOURAGE score instead of APACHE II score in the CVD group, the relationship between CFB and 90-day mortality remained significant. This relationship was also maintained when adjustments were made for PRESERVE score instead of APACHE II score in patients who had undergone VV-ECMO in the non-CVD group (Additional file 1: Table S3).

\section{Significant CFB threshold level in ECMO patients}

To further investigate the CFB threshold level that considerably increases the risk of mortality in ECMO patients, the relationship between CFB and mortality risk was evaluated by cubic spline analyses. In the CVD group, the relative $H R$ of mortality started to increase significantly when CFB values were greater than $82.3 \mathrm{ml} / \mathrm{kg}$ (Fig. 3a). The relative HR increased significantly above CFB levels of $189.6 \mathrm{ml} / \mathrm{kg}$ in the respiratory group (Fig. 3b). In the others group, relative HR tended to increase with higher CFB amounts without statistical significance (Fig. 3c).

\section{Discussion}

Large amounts of fluid resuscitation are inevitable in patients initiating ECMO. However, excessive fluid overload can negatively impact outcomes in these patients. In this study, we have shown that CFB was a significant factor affecting mortality risk in patients treated with ECMO. This increase in mortality risk was significant only when the CFB was greater than $82.3 \mathrm{ml} / \mathrm{kg}$ in patients who were treated with ECMO for cardiovascular reasons. For those who were treated with ECMO for respiratory causes, mortality risk increase was substantial when CFB was above $189.6 \mathrm{ml} / \mathrm{kg}$.

In patients undergoing ECMO treatment, large-volume fluid administration is frequently required. This is due mainly to disease severity. However, maintaining adequate blood flow for ECMO treatment also plays a part. Patients typically go through a systemic inflammatory response within the first few days of ECMO treatment [6]. This response induces pathologic vasodilation and fluid loss to the interstitial compartment, resulting in reduced vascular volume. In addition, major conditions associated with patients undergoing ECMO, such as shock and low cardiac output as well as increased capillary leakage related to sepsis-like syndrome, are factors that contribute $[8,32,33]$. This insufficient intravascular volume can lead to extracorporeal flow failure, which results in more frequent ECMO circuit changes and decreased total ECMO delivery time $[6,34]$. In a retrospective study of pediatric patients who received ECMO treatment for severe pneumonia, frequent circuit change was a significant risk factor for death [35]. In addition, in a recent investigation of 172 adult patients receiving ECMO, ECMO circuit change was more frequent in patients undergoing concomitant CRRT and ECMO treatment, which resulted in an increased mortality rate in these patients [19]. Therefore, the administration of

Table 4 Cox regression analyses for 90-day mortality

\begin{tabular}{|c|c|c|c|c|c|c|c|c|}
\hline \multirow{3}{*}{$\begin{array}{l}\text { CFB } \\
\text { quartiles }\end{array}$} & \multicolumn{4}{|c|}{ Cardiovascular disease } & \multicolumn{4}{|c|}{ Non-cardiovascular disease } \\
\hline & \multicolumn{2}{|l|}{ Unadjusted } & \multicolumn{2}{|l|}{ Adjusted* } & \multicolumn{2}{|l|}{ Unadjusted } & \multicolumn{2}{|l|}{ Adjusted* } \\
\hline & $\mathrm{HR}(95 \% \mathrm{Cl})$ & $P$ value & $\mathrm{HR}(95 \% \mathrm{Cl})$ & $P$ value & $\mathrm{HR}(95 \% \mathrm{Cl})$ & $P$ value & $\mathrm{HR}(95 \% \mathrm{Cl})$ & $P$ value \\
\hline Quartile 1 & 1.00 (reference) & & 1.00 (reference) & & 1.00 (reference) & & 1.00 (reference) & \\
\hline Quartile 2 & $1.17(0.75-1.83)$ & 0.496 & $1.27(0.77-2.10)$ & 0.342 & $0.84(0.56-1.27)$ & 0.407 & $0.79(0.48-1.30)$ & 0.345 \\
\hline Quartile 3 & $2.82(1.88-4.23)$ & $<0.001$ & $2.58(1.62-4.11)$ & $<0.001$ & $1.61(1.11-2.34)$ & 0.012 & $1.66(1.06-2.59)$ & 0.026 \\
\hline Quartile 4 & $2.24(1.47-3.41)$ & $<0.001$ & $2.11(1.26-3.54)$ & 0.004 & $1.37(0.93-2.02)$ & 0.108 & $1.69(1.05-2.72)$ & 0.030 \\
\hline
\end{tabular}

CFB Cumulative fluid balance

*Adjusted for age, sex, Charlson comorbidity index, Acute Physiology and Chronic Health Evaluation II score, and propensity score

Propensity score was obtained by logistic regression analysis with covariables body mass index, extracorporeal membrane oxygenation (ECMO) pump time, ECMO blood flow rate, albumin, total carbon dioxide, acute kidney injury stage

The 27 (3.7\%) patients who were lost to follow-up were treated as censored 
Table 5 Cox regression analyses for 90-day mortality with cumulative fluid balance, cumulative input, and output

\begin{tabular}{|c|c|c|c|c|c|}
\hline \multirow[t]{2}{*}{ Models } & \multirow[t]{2}{*}{ Variables } & \multicolumn{2}{|c|}{ Cardiovascular disease } & \multicolumn{2}{|c|}{ Non-cardiovascular disease } \\
\hline & & $\mathrm{HR}(95 \% \mathrm{Cl})$ & $P$ value & $\mathrm{HR}(95 \% \mathrm{Cl})$ & $P$ value \\
\hline Model 1 & $\mathrm{CFB}^{*}$ & $1.76(1.37-2.27)$ & $<0.001$ & $1.46(1.17-1.83)$ & $<0.001$ \\
\hline Model 2 & $\mathrm{CFB}^{*}$ & $1.67(1.19-2.34)$ & 0.003 & $1.55(1.16-2.09)$ & 0.003 \\
\hline \multirow[t]{2}{*}{ Model 3} & Cumulative input ${ }^{*}{ }^{\dagger}$ & $3.35(1.64-6.83)$ & 0.001 & $5.53(2.60-11.75)$ & $<0.001$ \\
\hline & Cumulative total output ${ }^{*, \dagger}$ & $0.56(0.47-0.76)$ & $<0.001$ & $0.25(0.14-0.45)$ & $<0.001$ \\
\hline \multirow[t]{2}{*}{ Model 4} & Cumulative input*,t & $2.66(1.30-5.44)$ & 0.007 & $1.92(1.08-3.42)$ & 0.027 \\
\hline & Cumulative urine output ${ }^{*},+$ & $0.78(0.67-0.91)$ & 0.001 & $0.84(0.75-0.93)$ & 0.001 \\
\hline
\end{tabular}

CFB Cumulative fluid balance

Model 1: Additionally adjusted for age, sex, Charlson comorbidity index, Acute Physiology and Chronic Health Evaluation II score, and propensity score Model 2: Model $1+$ daily fluid balance before ECMO ${ }^{*, 9}$

Model 3: Model $2+$ cumulative input + cumulative total output without CFB

Model 4: Model $2+$ cumulative input + cumulative urine output without CFB

*Data were log-transformed

${ }^{\dagger}$ During 3 days from extracorporeal membrane oxygenation (ECMO) commencement

"Daily fluid balance during intensive care unit admission before ECMO commencement

Propensity score was obtained by logistic regression analysis with covariables body mass index, ECMO pump time, ECMO blood flow rate, albumin, total carbon dioxide, acute kidney injury stage

The 27 (3.7\%) patients who were lost to follow-up were treated as censored

sufficient amounts of intravenous fluid to maintain a satisfactory extracorporeal blood flow is a critical component in the management of patients initiating ECMO. Accordingly, in this study, $85.9 \%$ of the patients maintained a positive CFB. In addition, the overall average CFB was greater than that of previously reported ICU patients not undergoing ECMO treatment [15, 21].

Excessive CFB during the initial phase of ECMO was found to be independently associated with increased mortality risk. This association between positive fluid balance and survival in ECMO-treated patients has been proposed previously. In a study of 172 patients receiving VA-ECMO or VV-ECMO treatment, positive fluid balance at the third day of ECMO treatment was an independent predictor of mortality [19]. In addition, in pediatric patients requiring concomitant ECMO and CRRT treatment, the degree of fluid overload at the time of ECMO initiation was significantly associated with mortality [36]. In this study, the CFB showed a significant independent relationship with 90 -day mortality. However, interestingly, the risk of mortality did not show a significant increase at CFB levels less than $82.3 \mathrm{ml} / \mathrm{kg}$ and $189.6 \mathrm{ml} / \mathrm{kg}$ for the CVD and respiratory groups, respectively. This finding suggests that patients undergoing ECMO might be able to tolerate fluid replacement below these thresholds. The risk of mortality was elevated in proportion to increases in the CFB above these thresholds, thereby confirming the relationship between CFB and mortality risk suggested in previous investigations $[19,20]$. However, the relationships between mortality and CFB were not linear. As mentioned above, decreased intravascular volume associated with inadequate fluid resuscitation could cause frequent ECMO circuit change, consequently affecting outcome [6,34].
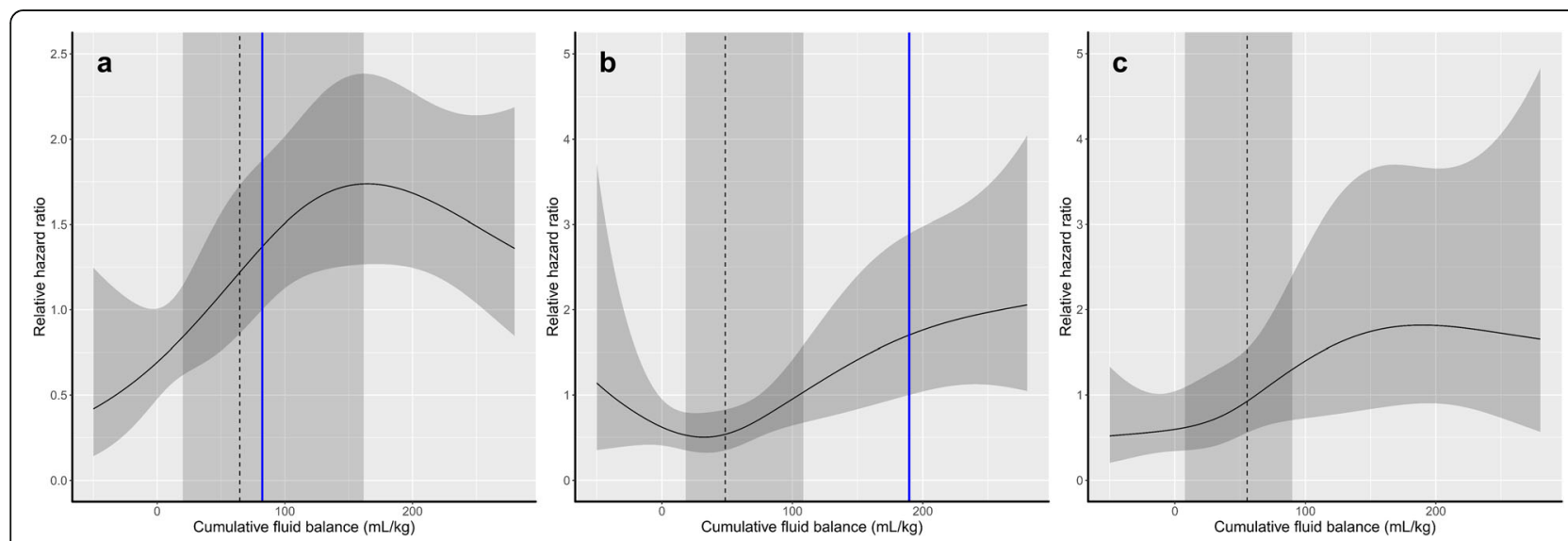

Fig. 3 Cubic spline curves for the association between cumulative fluid balance and 90-day mortality. a Cubic spline curve representing patients with cardiovascular disease. $\mathbf{b}$ Cubic spline curve representing patients with respiratory disease. c Cubic spline curve representing patients with other disease. Dashed line = median cumulative fluid balance, dark gray area =IQR, blue line $=$ permissive threshold of cumulative fluid balance 
This may be one of the reasons why mortality risk did not show a linear relationship at lower CFB levels. In the CVD group, HR of mortality did not seem to increase linearly when CFB was greater than $150 \mathrm{ml} / \mathrm{kg}$. However, the range of the CIs was too wide to infer a significant relationship. This could be due to the fact that the number of patients with extremely high CFB was relatively small. The threshold CFB amounts proposed in this study require further validation. However, in light of the fact that a positive fluid balance during the initial phase of ECMO treatment is generally unavoidable, the CFB thresholds suggested herein, as determined by mortality risk, could serve as a basis for the definition of the clinically significant fluid overload threshold in ECMO-treated patients.

The increased CFB in this study could have been a result of disease severity, considering that those with severe systemic vascular leakage and decreased cardiac function would need larger amounts of fluid resuscitation. The APACHE II scores were not significantly different between CFB quartiles in the CVD group. However, an APACHE II score increase was noticed in the highest quartile of the non-CVD group. Fluid overload may be a result of severe patient conditions, but it could also affect outcome by aggravating vascular wall stretching and worsening vascular permeability [37]. Because fluid balance and patient severity are interconnected during the treatment course of critically ill patients, it is not easy to delineate a causal relationship [37]. Accordingly, in the current study, owing to the retrospective nature of the investigation, defining the exact causality between severity and fluid balance was not possible. However, even after adjustments were made for disease severity, a significant increased risk of mortality was noted in the higher CFB quartiles, suggesting that fluid balance could have played an independent role in affecting outcome. Additionally, the fact that no significant interactions were found between disease severity and fluid balance further suggests the possibility that the results presented in this study were an effect of fluid balance rather than disease severity. Nevertheless, further investigations controlling the resuscitation volume amount would be needed to further confirm the findings of this study.

Both decreased output and increased input played a role in increasing CFB in ECMO-treated patients in this study. Lower cumulative total output and cumulative urine output were both independently associated with increased risk of mortality. Meanwhile, higher cumulative input also had a significant relationship with mortality. Notably, this significant relationship with cumulative input was robust even after adjustments were made for cumulative total output or cumulative urine output. Therefore, although decreased output contributes to the positive $\mathrm{CFB}$, increased fluid input still plays a significant role in affecting outcome. Moreover, cumulative input clearly increases the risk of mortality, regardless of output amount, suggesting that the positive input may affect outcome independently of disease severity.

The CFB threshold levels found in this study were higher in the non-CVD group than in the CVD group. The fact that the patients in the non-CVD group were younger and had lower APACHE II scores than those in the CVD group opens the possibility that the non-CVD group could have been healthier and thereby able to tolerate larger amounts of CFB than the CVD group. Hemodynamic status in VV-ECMO-receiving patients is determined by a complex interaction of factors, such as intrathoracic pressure, native cardiac function, pulmonary vascular resistance, systemic vascular tone, and recirculation on VV-ECMO, in addition to intravascular volume. However, the effects of intrathoracic pressure, native cardiac function, and pulmonary vascular resistance on maintaining hemodynamic status are not substantial in patients undergoing VA-ECMO, making these patients relatively more vulnerable to volume than VV-ECMO patients. Furthermore, in VA-ECMO patients, unloading the heart with strict volume control may result in effective preload reduction. However, in VV-ECMO patients, an adequate amount of preload would be needed to maintain cardiac output. This could be one of the reasons that the permissive CFB amount was lower in the CVD group, in which most of the patients underwent VA-ECMO, than in the non-CVD group. Considering that most of the patients in the CVD group underwent VA-ECMO, ECMO modality could also have played a role in determining CFB threshold levels.

There are several limitations of this study. First, although adjustments were made for various confounding factors, including those that reflected the disease severity of the patients and the propensity scores, the limitations owing to the retrospective design should still be recognized. Second, data regarding medications such as diuretics, and hemodynamic parameters including pulmonary arterial pressure, cardiac chamber size, and cardiac output were not obtainable. Third, parameters to ensure sufficient ECMO treatment, such as oxygen consumption, RBC transfusion, amount of recirculation, incidence of catheter change, or ECMO circuit change could not be retrieved from the electronic medical records. Fourth, although protocol-based treatments were applied for all patients within each center, protocol details and bedside care would have varied somewhat from center to center and also over time. Finally, because the indications and modes of ECMO treatment varied, the results of this study may not be generalizable to all patients undergoing ECMO. Although the results were 
controlled for different indications and ECMO modalities, validation in specific subgroups is needed.

\section{Conclusions}

Excessive CFB during the early phase of ECMO treatment increased the risk of mortality. However, this risk did not increase significantly until the CFB reached specific threshold ranges, which differed according to the disease origin responsible for ECMO commencement. These results suggest that fluid therapy in ECMO-treated patients should be adjusted so as not to exceed this clinically significant fluid overload level. Further interventional studies are needed to confirm these findings.

\section{Additional files}

Additional file 1: Table S1. ECMO protocols for patients. Table S2. Baseline characteristics of patients with respiratory disease in non-CVD group. Table S3. Cox regression analyses for 90-day mortality with ENCOURAGE and PRESERVE scores. (DOCX $31 \mathrm{~kb}$ )

Additional file 2: Figure S1. Cumulative fluid balance according to ECMO treatment vintage. (PDF $31 \mathrm{~kb}$ )

\section{Abbreviations \\ ACT: Activated clotting time; AKI: Acute kidney injury; APACHE: Acute Physiology and Chronic Health Evaluation; ARDS: Acute respiratory distress syndrome; AT: Antithrombin; BMI: Body mass index; CFB: Cumulative fluid balance; CRRT: Continuous renal replacement therapy; CVD: Cardiovascular disease; ECMO: Extracorporeal membrane oxygenation; eGFR: Estimated glomerular filtration rate; ENCOURAGE: Prediction of cardiogenic shock outcome for AMI patients salvaged; ESRD: End-stage renal disease; ICU: Intensive care unit; LOS: Length of stay; PRESERVE: Predicting death for Severe ARDS on W-ECMO; RBC: Red blood cell; VA: Venoarterial; W: Venovenous}

\section{Acknowledgements}

This work was supported by the Soonchunhyang University Research Fund and a grant of the Korea Health Technology R\&D Project through the Korea Health Industry Development Institute (KHIDI), funded by the Ministry of Health \& Welfare, Republic of Korea (grant number HI17C1827).

\section{Availability of data and materials}

The data that support the findings of this study are available from the corresponding author upon reasonable request.

\section{Authors' contributions}

HK, JHS, HL, SHH, THY, SWK, SK, and JTP made substantial contributions to the conception and design of this study. HK, JHP, SP, JHJ, HRY, and YKK made contributions to acquisition and interpretation of data. HK, JTP, and SK drafted the manuscript and revised it for important intellectual content. All authors read and provided final approval of the version to be published and agreed to be accountable for all aspects of the work in ensuring that questions related to the accuracy or integrity of any part of the work are appropriately investigated and resolved.

\section{Ethics approval and consent to participate}

The study protocol was approved by the institutional review boards of Yonsei University Health System and Seoul National University Bundang Hospital. Informed consent was waived by the institutional review boards, owing to the retrospective study design.

\section{Consent for publication}

Not applicable.

\section{Competing interests}

The authors declare that they have no competing interests.

\section{Publisher's Note}

Springer Nature remains neutral with regard to jurisdictional claims in published maps and institutional affiliations.

\section{Author details}

${ }^{1}$ Division of Nephrology, Soonchunhyang University Seoul Hospital, Seoul, Republic of Korea. ${ }^{2}$ Department of Internal Medicine, Institute of Kidney Disease Research, Yonsei University College of Medicine, 50-1 Yonsei-ro, Seodaemun-Gu, Seoul 03722, Republic of Korea. ${ }^{3}$ Department of Internal Medicine, Seoul National University Bundang Hospital, 82, Gumi-ro 173 Beon-gil, Bundang-gu, Seongnam, Gyeonggi-do 13620, Republic of Korea. ${ }^{4}$ Department of Internal Medicine, Institute of Chest Disease, Yonsei University, Seoul, Republic of Korea. ${ }^{5}$ Department of Internal Medicine, Seoul National University College of Medicine, Seoul, Republic of Korea. ${ }^{6}$ Division of Nephrology and Hypertension, Department of Internal Medicine, Inha University College of Medicine, Incheon, Republic of Korea. ${ }^{7}$ Department of Internal Medicine, Hangang Sacred Heart Hospital, Hallym University College of Medicine, Seoul, Republic of Korea.

Received: 26 April 2018 Accepted: 25 September 2018

Published online: 27 October 2018

\section{References}

1. Peek GJ, Mugford M, Tiruvoipati R, Wilson A, Allen E, Thalanany MM, et al. Efficacy and economic assessment of conventional ventilatory support versus extracorporeal membrane oxygenation for severe adult respiratory failure (CESAR): a multicentre randomised controlled trial. Lancet. 2009;374: 1351-63.

2. Brodie D, Bacchetta M. Extracorporeal membrane oxygenation for ARDS in adults. N Engl J Med. 2011;365:1905-14.

3. Rastan AJ, Dege A, Mohr M, Doll N, Falk V, Walther T, et al. Early and late outcomes of 517 consecutive adult patients treated with extracorporeal membrane oxygenation for refractory postcardiotomy cardiogenic shock. J Thorac Cardiovasc Surg. 2010;139:302-11.e1.

4. Mosier JM, Kelsey M, Raz Y, Gunnerson KJ, Meyer R, Hypes CD, et al. Extracorporeal membrane oxygenation (ECMO) for critically ill adults in the emergency department: history, current applications, and future directions. Crit Care. 2015;19:431.

5. Yap HJ, Chen YC, Fang JT, Huang CC. Combination of continuous renal replacement therapies (CRRT) and extracorporeal membrane oxygenation (ECMO) for advanced cardiac patients. Ren Fail. 2003;25:183-93.

6. Sidebotham D, McGeorge A, McGuinness S, Edwards M, Willcox T, Beca J. Extracorporeal membrane oxygenation for treating severe cardiac and respiratory failure in adults: part 2-technical considerations. J Cardiothorac Vasc Anesth. 2010;24:164-72.

7. Sidebotham D. Troubleshooting adult ECMO. J Extra Corpor Technol. 2011; 43:P27-32.

8. Heradstveit BE, Guttormsen AB, Langorgen J, Hammersborg SM, WentzelLarsen T, Fanebust R, et al. Capillary leakage in post-cardiac arrest survivors during therapeutic hypothermia - a prospective, randomised study. Scand J Trauma Resusc Emerg Med. 2010;18:29.

9. Bernard GR, Artigas A, Brigham KL, Carlet J, Falke K, Hudson L, et al. American-European Consensus Conference on ARDS. Definitions, mechanisms, relevant outcomes, and clinical trial coordination. Am J Respir Crit Care Med. 1994;149:818-24.

10. Cheng R, Hachamovitch R, Kittleson M, Patel J, Arabia F, Moriguchi J, et al. Complications of extracorporeal membrane oxygenation for treatment of cardiogenic shock and cardiac arrest: a meta-analysis of 1,866 adult patients. Ann Thorac Surg. 2014;97:610-6.

11. Zangrillo A, Landoni G, Biondi-Zoccai G, Greco M, Greco T, Frati G, et al. A meta-analysis of complications and mortality of extracorporeal membrane oxygenation. Crit Care Resusc. 2013;15:172-8.

12. Brogan TV, Thiagarajan RR, Rycus PT, Bartlett RH, Bratton SL. Extracorporeal membrane oxygenation in adults with severe respiratory failure: a multicenter database. Intensive Care Med. 2009:35:2105-14.

13. Lee SW, Yu MY, Lee H, Ahn SY, Kim S, Chin HJ, et al. Risk factors for acute kidney injury and in-hospital mortality in patients receiving extracorporeal membrane oxygenation. PLoS One. 2015;10:e0140674. 
14. Askenazi DJ, Selewski DT, Paden ML, Cooper DS, Bridges BC, Zappitelli M, et al. Renal replacement therapy in critically ill patients receiving extracorporeal membrane oxygenation. Clin J Am Soc Nephrol. 2012;7:1328-36.

15. Neyra JA, Li X, Canepa-Escaro F, Adams-Huet B, Toto RD, Yee J, et al. Cumulative fluid balance and mortality in septic patients with or without acute kidney injury and chronic kidney disease. Crit Care Med. 2016;44: 1891-900.

16. Vincent JL, Sakr Y, Sprung CL, Ranieri VM, Reinhart K, Gerlach H, et al. Sepsis in European intensive care units: results of the SOAP study. Crit Care Med. 2006:34:344-53.

17. Micek ST, McEvoy C, McKenzie M, Hampton N, Doherty JA, Kollef MH. Fluid balance and cardiac function in septic shock as predictors of hospital mortality. Crit Care. 2013;17:R246

18. Kim F, Nichol G, Maynard C, Hallstrom A, Kudenchuk PJ, Rea T, et al. Effect of prehospital induction of mild hypothermia on survival and neurological status among adults with cardiac arrest: a randomized clinical trial. JAMA. 2014;311:45-52.

19. Schmidt M, Bailey M, Kelly J, Hodgson C, Cooper DJ, Scheinkestel C, et al. Impact of fluid balance on outcome of adult patients treated with extracorporeal membrane oxygenation. Intensive Care Med. 2014;40:1256-66.

20. Staudacher DL, Gold W, Biever PM, Bode C, Wengenmayer T. Early fluid resuscitation and volume therapy in venoarterial extracorporeal membrane oxygenation. J Crit Care. 2016;37:130-5.

21. Wang N, Jiang L, Zhu B, Wen Y, Xi XM, Beijing Acute Kidney Injury Trial Workgroup. Fluid balance and mortality in critically ill patients with acute kidney injury: a multicenter prospective epidemiological study. Crit Care. 2015;19:371.

22. Sakr Y, Rubatto Birri PN, Kotfis K, Nanchal R, Shah B, Kluge S, et al. Higher fluid balance increases the risk of death from sepsis: results from a large international audit. Crit Care Med. 2017:45:386-94.

23. Rosenberg AL, Dechert RE, Park PK, Bartlett RH, NHI NHBLI ARDS Network. Review of a large clinical series: association of cumulative fluid balance on outcome in acute lung injury: a retrospective review of the ARDSnet tidal volume study cohort. J Intensive Care Med. 2009;24:35-46.

24. Roch A, Guervilly C, Papazian L. Fluid management in acute lung injury and ARDS. Ann Intensive Care. 2011:1:16.

25. de Almeida JP, Palomba H, Galas FR, Fukushima JT, Duarte FA, Nagaoka D, et al. Positive fluid balance is associated with reduced survival in critically ill patients with cancer. Acta Anaesthesiol Scand. 2012;56:712-7.

26. Muller $G$, Flecher $E$, Lebreton $G$, Luyt CE, Trouillet JL, Brechot N, et al. The ENCOURAGE mortality risk score and analysis of long-term outcomes after VA-ECMO for acute myocardial infarction with cardiogenic shock. Intensive Care Med. 2016;42:370-8.

27. Schmidt M, Zogheib E, Roze H, Repesse X, Lebreton G, Luyt CE, et al. The PRESERVE mortality risk score and analysis of long-term outcomes after extracorporeal membrane oxygenation for severe acute respiratory distress syndrome. Intensive Care Med. 2013;39:1704-13.

28. Levey AS, Bosch JP, Lewis JB, Greene T, Rogers N, Roth D, Modification of Diet in Renal Disease Study Group. A more accurate method to estimate glomerular filtration rate from serum creatinine: a new prediction equation. Ann Intern Med. 1999;130:461-70.

29. KDIGO AKI Work Group. KDIGO clinical practice guideline for acute kidney injury. Kidney Int Suppl. 2012;17:1-138.

30. Wi J, Noh H, Min KL, Yang S, Jin BH, Hahn J, et al. Population pharmacokinetics and dose optimization of teicoplanin during venoarterial extracorporeal membrane oxygenation. Antimicrob Agents Chemother. 2017;61(9):e01015-7.

31. Canaud B, Barbieri C, Marcelli D, Bellocchio F, Bowry S, Mari F, et al. Optimal convection volume for improving patient outcomes in an international incident dialysis cohort treated with online hemodiafiltration. Kidney Int. 2015;88:1108-16

32. Hou G, Yu K, Yin X, Wang H, Xu W, Du Z, et al. Safety research of extracorporeal membrane oxygenation treatment on cardiogenic shock: a multicenter clinical study. Minerva Cardioangiol. 2016;64:121-6.

33. Adrie C, Adib-Conquy M, Laurent I, Monchi M, Vinsonneau C, Fitting C, et al. Successful cardiopulmonary resuscitation after cardiac arrest as a "sepsislike" syndrome. Circulation. 2002;106:562-8.

34. Lubnow M, Philipp A, Foltan M, Bull Enger T, Lunz D, Bein T, et al. Technical complications during veno-venous extracorporeal membrane oxygenation and their relevance predicting a system-exchange - retrospective analysis of 265 cases. PLoS One. 2014;9:e112316.
35. Smalley N, MacLaren G, Best D, Paul E, Butt W. Outcomes in children with refractory pneumonia supported with extracorporeal membrane oxygenation. Intensive Care Med. 2012;38:1001-7.

36. Selewski DT, Cornell TT, Blatt NB, Han YY, Mottes T, Kommareddi M, et al. Fluid overload and fluid removal in pediatric patients on extracorporeal membrane oxygenation requiring continuous renal replacement therapy. Crit Care Med. 2012;40:2694-9.

37. Ostermann M, Straaten HM, Forni LG. Fluid overload and acute kidney injury: cause or consequence? Crit Care. 2015;19:443.
Ready to submit your research? Choose BMC and benefit from:

- fast, convenient online submission

- thorough peer review by experienced researchers in your field

- rapid publication on acceptance

- support for research data, including large and complex data types

- gold Open Access which fosters wider collaboration and increased citations

- maximum visibility for your research: over $100 \mathrm{M}$ website views per year

At BMC, research is always in progress.

Learn more biomedcentral.com/submissions 